\title{
Next-generation materials for future synchrotron and free-electron laser sources
}

\author{
Lahsen Assoufid and Heinz Graafsma, Guest Editors
}

\begin{abstract}
The development of new materials and improvements of existing ones are at the root of the spectacular recent developments of new technologies for synchrotron storage rings and free-electron laser sources. This holds true for all relevant application areas, from electron guns to undulators, x-ray optics, and detectors. As demand grows for more powerful and efficient light sources, efficient optics, and high-speed detectors, an overview of ongoing materials research for these applications is timely. In this article, we focus on the most exciting and demanding areas of materials research and development for synchrotron radiation optics and detectors. Materials issues of components for synchrotron and freeelectron laser accelerators are briefly discussed. The articles in this issue expand on these topics.
\end{abstract}

\section{Introduction}

$\mathrm{X}$-ray synchrotron radiation and free-electron laser (XFEL) sources (also referred to collectively as X-ray light sources) are important tools in materials science for probing the atomic and electronic structure of materials and their surfaces, as well as the dynamics of atomic- and molecularlevel processes. ${ }^{1}$ In turn, materials research is critical for the advancement of the fundamental technologies underpinning these sources. In this issue of MRS Bulletin, we explore recent advances in materials for the optics, detectors, and other components that are essential parts of these research tools.

$\mathrm{X}$-ray synchrotron radiation is generated by a beam of electrons (or positrons) that are accelerated to nearly the speed of light. While passing through a linear periodic magnetic field created by specialized magnet devices (or insertion devices) called undulators, these electrons (or positrons) oscillate and emit x-rays at each bend. X-rays emitted at consecutive bends add up to form a powerful laser-like, quasi-monochromatic $\mathrm{x}$-ray beam billions of times brighter ${ }^{2,3}$ than light from conventional laboratory x-ray tubes (Figure 1), that can probe matter at the atomic scale. In addition to brightness, these $\mathrm{x}$-ray light sources exhibit natural collimation, which leads to greatly improved resolution, as well as the ability to select a single photon energy that interacts with the material of interest, yielding an optimum signal-to-noise ratio. These characteristics have enabled detailed studies ranging from materials behavior and properties under extreme conditions ${ }^{4}$ (Figure 2) to the behavior of biological systems at the cellular and molecular levels. Experiments at synchrotron sources have resulted in important discoveries, as evidenced by the Nobel prizes received for such work.

$\mathrm{X}$-ray sources have seen an exponential improvement over the last 40-50 years, a trend that is continuing today (Figure 1). New XFEL sources are being developed worldwide that promise high repetition rates with unprecedented brilliance (also called spectral brightness or simply, brightness, which is the number of photons $/$ second $/$ millimeter $^{2} /$ milliradian $^{2} / 0.1 \%$ energy bandwidth), and pico- to femtosecond timing over a broad photon energy range with full, or a high degree of, spatial and temporal coherence ${ }^{5}$ these sources will enable revolutionary advances in x-ray science and new discoveries in the fields of physics, biology, materials science, and engineering. Also, "ultimate" storage rings will allow powerful new experiments that take advantage of the full coherence and brightness of the diffraction-limited radiation.

These new sources present major challenges and new opportunities to develop advanced optical elements and detectors. All optical elements in the beam path must be manufactured to a high degree of precision, and their materials must maintain their characteristics during use to preserve the integrity of the source brightness. The detector materials 

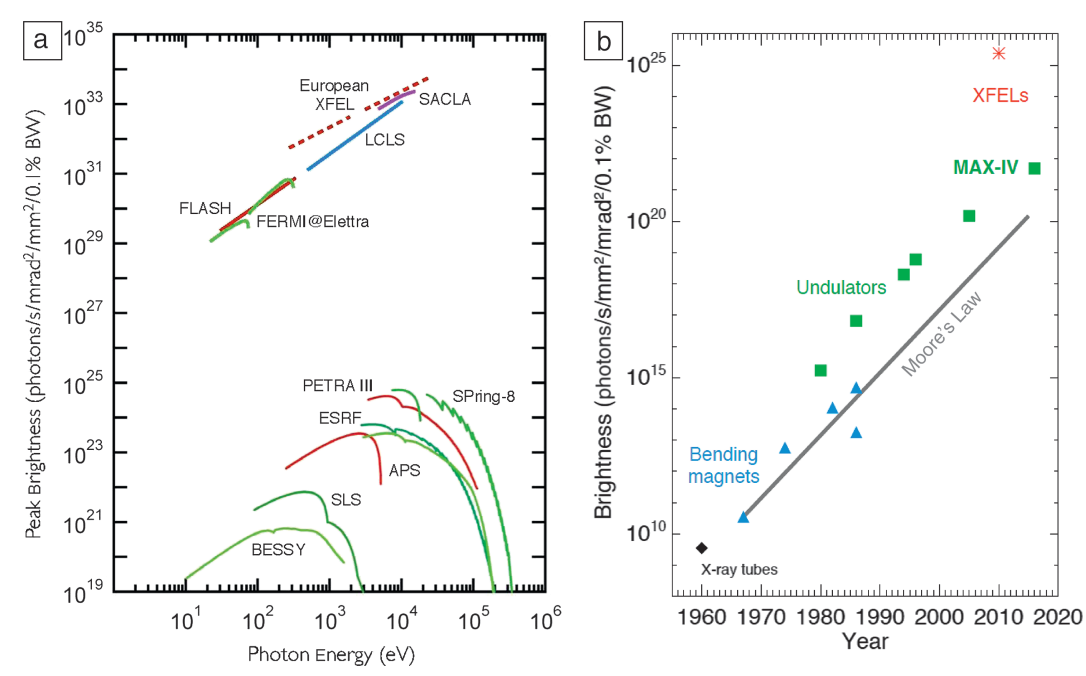

Figure 1. Comparison of different $x$-ray source devices and facilities. (a) Peak brightness of synchrotrons and free-electron lasers worldwide. ${ }^{2}$ (b) Average brightness of $x$-ray tubes, bending (dipole) magnet sources at storage rings, undulators (linear periodic magnet) devices at storage rings, $\mathrm{x}$-ray free-electron lasers (XFELs), and MAX-IV-the newly built diffraction-limited source at the MAX-IV lab in Sweden. ${ }^{3}$

\section{Materials challenges for x-ray optics}

The $\mathrm{x}$-ray beams produced by modern synchrotron sources provide enormous capabilities; however, further conditioning is required to match the needs of the experiments. To produce incident beams with the required wavelength, monochromaticity or bandwidth, spot size, polarization, and intensity, various $\mathrm{x}$-ray optical elements are utilized. After the beam has interacted with the sample, x-ray optics are often used to collect or disperse the resulting photons before detection. Optics used in $\mathrm{x}$-ray light sources can be grouped in three major categories - mirrors, monochromators, and lenses (or focusing elements).

$\mathrm{X}$-rays interact with matter differently compared to visible light because of their high penetration depth and low index of refraction $(<1)$. These properties impose special requirements for x-ray optics, and these requirements will become more severe with increasing coherence lengths foreseen for the new generation of storage rings.

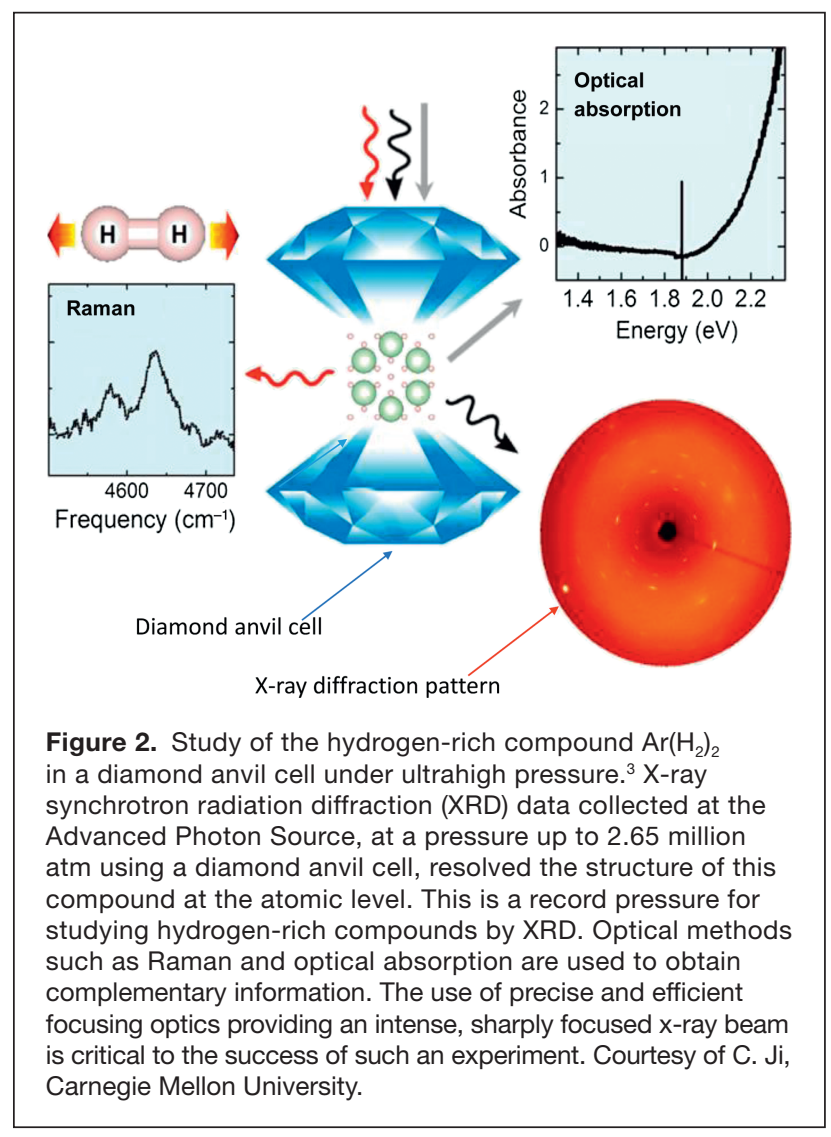

must be efficient and able to withstand a high photon flux. Improvements in both detectors and optics highly depend on advances in specialized materials as well as in the availability of customized fabrication techniques.
Materials for x-ray optics must have high radiation resistance, long-term stability, and excellent thermal-mechanical properties (low thermal expansion coefficients and high thermal conductivities). They should be available in large sizes, and they must be easy to fabricate, machine, and polish. Also, the x-ray optics used for beam conditioning are generally located in an ultrahigh vacuum environment, so interventions for repair or adjustments cause significant downtimes for experiments. Since synchrotrons are expensive to run, and experimental time is precious and difficult to obtain, $\mathrm{x}$-ray optics must be as reliable as possible.

Each optical element has its own specific challenges related to the nature of the interaction with the x-ray beam (i.e., reflection, diffraction, or refraction). In this article, we briefly touch upon a few aspects of materials of the most important optical elements in x-ray light source beamlines, namely mirrors, monochromators, and focusing optics.

Mirrors reflect the x-rays by total reflection at a low grazing incidence angle, typically a few tenths of a degree, from the mirror's reflecting surface, and are used for a variety of functions, including low-pass filtering, collimation, steering, harmonic rejection, and focusing ${ }^{6}$ (Figure 3). As a result, $\mathrm{x}$-ray mirrors have a rectangular cross section of a few centimeters $(\mathrm{cm})$ by a few $\mathrm{cm}$ and can be on the order of $1 \mathrm{~m}$ long to intercept the full incident beam footprint (typically $1 \mathrm{~mm} \times 1 \mathrm{~mm}$ at distances of tens of meters from the source) at a few milliradians incidence angle. To preserve the quality of the incident beam wavefront and provide maximum reflectivity, the substrate material must not only be polished to atomic-scale roughness, but must have a low thermal expansion coefficient and high thermal conductivity to minimize the mirror's thermomechanical distortions, ideally over a 


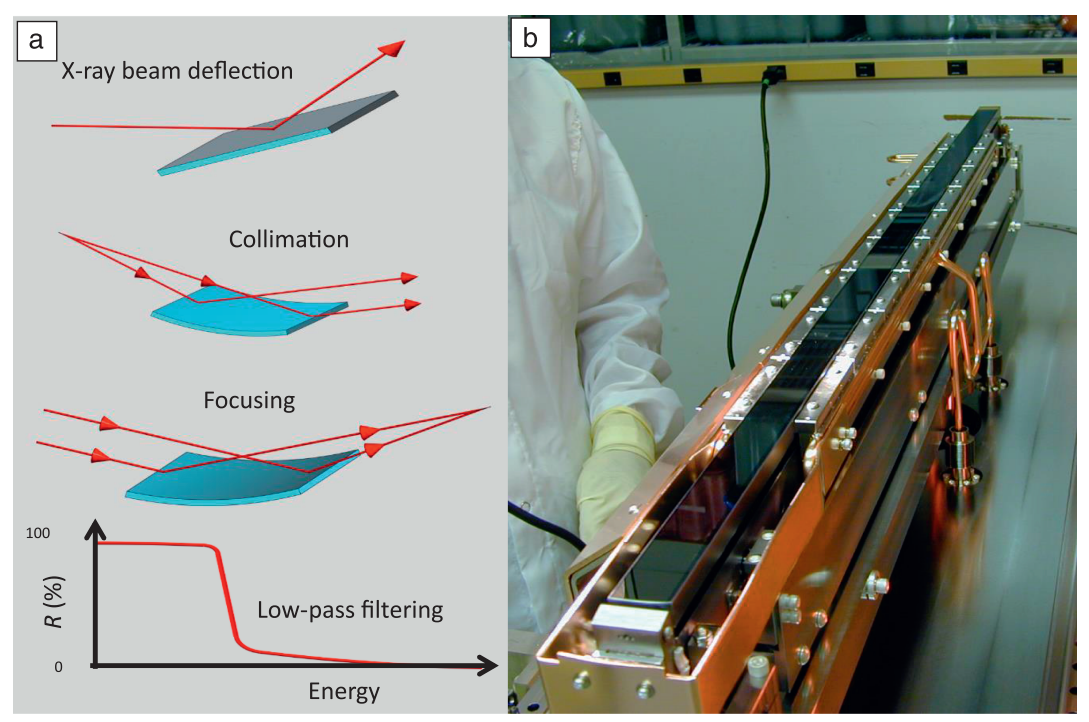

Figure 3. (a) Schematic of some of the functions served by mirrors as x-ray optical components. $^{6}$ (b) Example of a Si x-ray mirror ready for installation in a synchrotron beamline. Courtesy of T. Rabedeau, SLAC/SSRL, Stanford University. diffraction imaging, and small-angle x-ray scattering. The performance of these optics is strongly dependent on the quality and the compatibility of the bilayer materials, the interlayer roughness, the uniformity of the layers, and their stability. Examples of bilayer materials include $\mathrm{W} / \mathrm{C}, \mathrm{W} / \mathrm{Si}$, and $\mathrm{Mo} / \mathrm{Si}$. One of the biggest challenges is to produce low interfacial roughness ${ }^{8}$ with extremely low built-in stress. Therefore, the choice of bilayer materials and the ratio of the thicknesses of the layers are critical to achieving optimum performance. The use of periodic trilayer material structures has been shown to enhance reflectivity compared to a periodic bilayer material. ${ }^{9}$

The development of diffraction-limited synchrotron radiation storage rings (DLSRs) makes it possible to focus $\mathrm{x}$-rays to a nanoscale spot size, thus enabling new scientific research opportunities and imaging at a single-particle level. X-ray focusing is conducted through a variety of optical elements using diffraction, wide range of temperatures. Very few materials meet these demands. Silicon (Si) is the most used mirror substrate material. Its excellent thermal expansion coefficient, which is about $2.6 \cdot 10^{-6} / \mathrm{K}$ at room temperature, goes to zero at $\sim 124 \mathrm{~K}$ (or about $-150^{\circ} \mathrm{C}$ ), and its thermal conductivity is improved by an order of magnitude at liquid nitrogen temperature $\left(\sim 193^{\circ} \mathrm{C}\right)$ compared to room temperature. Others include ultralowexpansion glass, fused silica, and zerodur (a lithium aluminosilicate glass-ceramic produced by Schott AG). These materials have poor thermal conductivity compared to $\mathrm{Si}$, and some develop color centers under intense x-rays. XFEL beams are characterized by extreme brightness, ultrashort pulse duration, and high peak power, so radiation damage to the mirror's reflecting materials is a serious concern. ${ }^{7}$

Monochromators select the wavelength of interest for a particular experiment from the $\mathrm{x}$-ray beam and analyze the signal after the incident beam interacts with the sample as a consequence of the diffraction of the beam by the crystal lattice. The type of crystal material used depends on the wavelength and the bandwidth of the desired x-ray spectrum. Ideally, the materials should be of high crystalline purity and dislocationfree, and, for some applications, must provide a wide range of selectable reflections. In their article in this issue, Yavaş et al. provide a detailed discussion on monochromator materials and requirements for high-resolution applications.

In some cases, multilayer optics and reflection gratings are used for monochromatization. Multilayers are artificial onedimensional (1D) crystals made by sputtering a stack of $10 \mathrm{~s}$ or even 100s periodic bilayers of alternating low-density and high-density materials or alloys on a supersmooth substrate. They provide 100 -fold more bandwidth compared to crystal monochromators, which is important for flux-hungry experimental techniques such as phase-contrast imaging, coherent refraction, or reflection. Focusing optics present material challenges with respect to homogeneity and minimal absorption of radiation at the wavelength of interest. In their article in this issue, Roth et al. discuss refractive focusing lenses in detail. Here, we briefly highlight materials issues for two types of diffractive focusing optical element, Fresnel zone plates ${ }^{10}$ and multilayer Laue lenses (MLLs), ${ }^{11}$ which have the potential of achieving sub-10-nm spot size. A zone plate consists of a grating-like set of concentric rings, known as Fresnel zones, that alternate between opaque and transparent, with a decreasing width from the center to the edge of the optic. The spot size and the focusing efficiency are directly related to the width of the outermost zone relative to the thickness of the zone plate. For high-energy x-rays, aspect ratios of zone thickness to width of more than 100 are needed. Therefore, their fabrication requires advanced tools and materials. The MLL is a type of linear zone plate, made by thin-film sputtering, that offers unlimited aspect ratio with the potential of achieving sub-10-nm focusing and unmatched efficiency at very short $(<0.1 \mathrm{~nm})$ wavelengths. MLLs require depositing thousands of bilayer/ trilayer materials with graded thickness. Such an optic has achieved a record $15 \mathrm{~nm} \times 15 \mathrm{~nm}$ focus at $0.1 \mathrm{~nm}$ wavelength for routine experimental use. ${ }^{12}$ Intense research is underway worldwide to reach the ultimate focus.

\section{Materials challenges for $\mathbf{x}$-ray detectors}

At every experiment station in a synchrotron facility, there is an x-ray detector, and it is often the capabilities of this detector that determine the potential value of the experiment. New $\mathrm{x}$-ray sources pose a formidable challenge to the detector, not only for the electronics and signal processing parts, but also for the layer that absorbs the incoming x-ray photons. ${ }^{13}$ With increasing flux, these materials will need to be more 
radiation hard. With the large instantaneous fluxes generated by XFELs, these materials will require faster charge-transport properties and lower afterglow (where carriers are slowly released from traps, producing signal over a long time period after the original x-ray hit). With future DLSRs, there is a shift to higher photon energies, where silicon becomes increasingly inefficient, so new materials are required. With the small source sizes at DLSRs, even higher spatial and time resolutions are needed, requiring new scintillators. The vast variety of experiments and techniques that will be performed, each requiring different aspects to be optimized, makes materials science a fundamental part of detector science.

\section{Material challenges for linear and circular accelerators}

The continuing development of $\mathrm{x}$-ray light sources would greatly benefit from the availability of advanced materials such as high-temperature superconductors and new materials for insulators, as well as more efficient materials for electron guns and klystrons (i.e., high-frequency power sources). The availability of these materials will significantly shrink the size of magnets, undulators, and radio-frequency cavities and improve their performance.

The next generation of linear and circular particle beam accelerators based on novel approaches to accelerator design present major challenges, with a strong emphasis on smaller footprints and reduced capital and operation costs. Significant R\&D efforts are anticipated, with a focus on understanding the fundamental physics of the surfaces of the material and bulk properties, to increase the gradient of the accelerating field in both normal and superconducting structures beyond the current limits.

Normal-conducting copper has been widely used for radio-frequency cavities because of its excellent properties, even at room temperature. Ongoing and new R\&D programs will explore pushing the performance limits of copper by, for example, modifying its properties or operating it at cryogenic temperatures. A new approach to improve the performance of bulk niobium superconducting accelerating cavities has produced outstanding results. ${ }^{14}$ Novel medium- to high- $\mathrm{T}_{\mathrm{c}}$ superconducting materials such as $\mathrm{Nb}_{3} \mathrm{Sn}$ (niobium-tin) ${ }^{15}$ and $\mathrm{MgB}_{2}$ (magnesium diboride) ${ }^{16}$ thin films on niobium and copper structures are being investigated. An important goal is operation of the accelerating structures at a higher temperature than liquid helium temperature, resulting in significant cost savings.

\section{In this issue Optics}

Three articles in this issue focus on diamond optics, materials for high-energy-resolution optics, and materials for refractive lenses.

In their article, Shvyd'ko et al. review recent progress in synthesizing flawless diamond crystal components that exploit the material's outstanding advantages compared to silicon in radiation hardness, $\mathrm{x}$-ray transparency, stiffness, thermal conductivity, thermal-expansion properties, and reflectivity.

The article by Yavaş et al. provides an overview of crystal monochromators and analyzers and explores recent advances in materials beyond silicon for application to high-energy resolution $(\sim 10 \mathrm{meV}$ or better) techniques such as resonant inelastic x-ray scattering and nuclear resonant scattering.

The use of $x$-ray refractive lenses has quickly expanded to become mainstream in many applications at all light sources worldwide. However, the fabrication methods as well as the materials $(\mathrm{Be}, \mathrm{Al})$ commonly used in their fabrication still have many shortcomings in terms of their ability to produce scatter and preserve the beam wavefront. In their article, Roth et al. provide an excellent overview of the state of the art of both materials and fabrication methods for 1D and twodimensional focusing lenses and discuss ongoing developments and potential future directions.

\section{Detectors}

With exponential increases in the source brilliance of synchrotron storage-ring sources, and more recently, with the nine-orders-of-magnitude increase in peak brilliance of XFEL sources as compared to even the brightest storage rings, ${ }^{2}$ custom-designed detectors have become mandatory. A variety of such detectors and detector systems have been constructed (Figure 4) and commercialized in recent years. Most of these are based on hybrid-pixel detector technology, with silicon as the x-ray absorbing sensor. Silicon-based hybrid-pixel array detectors (HPADs) are perfectly suited for the photon energy range between approximately 3 and $20 \mathrm{keV}$, and spatial resolutions of a few tens of microns and up.

Increasingly important are photon energies greater than $20 \mathrm{keV}$, especially at DLSRs. This means that alternatives to silicon as the photon absorbing material are required. Considerable effort and resources have been invested worldwide over the last few decades in developing high- $Z$ semiconductor materials

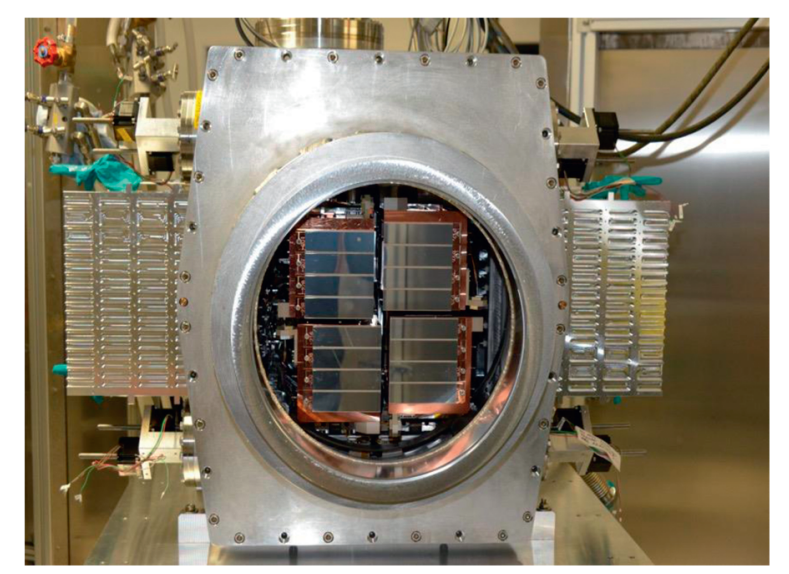

Figure 4. The adaptive gain integrating pixel detector, custom developed for the European X-ray Free Electron Laser facility in Hamburg, Germany. 
(e.g., CdTe, CdZnTe, and GaAs) for x-ray detection, especially for and by the medical imaging and homeland security markets. Much progress has been achieved in improving material quality as well as in sensor-processing technologies. Nevertheless, these materials remain relatively imperfect, especially under intense irradiation. Ge is an alternative to GaAs, but requires deep cooling because of the small bandgap, posing challenges in system designs. In their article, Pennicard et al. describe the current state, the remaining challenges, and the perspectives for the previously mentioned materials.

Another area not well addressed by silicon HPADs is imaging with micrometer or better spatial resolution. Here, the system of choice is a photon detector, generally a charge-coupled device or complementary metal oxide semiconductor imager, lens-coupled to an input scintillator. Whereas the photon detector and optical relay system are relatively straightforward, the choice of the right scintillator is less obvious. There are a wide variety of scintillators, each with its own advantages and disadvantages. Important parameters include light yield, emission spectrum, decay times, afterglow, and radiation hardness. In addition, substrate materials, ease of production, processing, and handling conditions determine usability of the materials. The article by Martin et al. gives a clear overview of the most commonly used scintillators and their applicability to $\mathrm{X}$-ray detectors at synchrotron and XFEL sources.

In addition to developments of x-ray absorbing and converting materials described in the two contributions on detectors, there are many developments and opportunities in the field of novel construction materials and fabrication processes, such as three-dimensional printing and carbon-based lightweight structures that are relevant or even crucial for modern detectors.

\section{Outlook}

New materials and novel processing techniques will undoubtedly play a central role in further advancing the key components of future diffraction-limited light sources, from electron gun technology to magnet and vacuum technologies. In turn, exploiting the full potential of these light sources will require near-perfect optics, highly stable beamlines and sample environments, and highly efficent and specialized detectors.

For optics, silicon will likely remain the material of choice for now for monochromators and mirror substrates for most $\mathrm{X}$-ray synchrotron radiation and XFEL techniques. New deterministic fabrication and processing techniques are expected to emerge to further improve the quality of both silicon- and nonsilicon-based optics. Development of materials beyond silicon will be key in developing more efficient and higherresolution optics.

Better and faster scintillators will likely appear over the coming years. The high- $Z$ semiconductors used today will see continuous improvement in quality and reliability, both in raw materials and in processing. It is less likely that new compound semiconductors will be used in x-ray detectors in the near future. What is very likely, on the other hand, is that novel materials and fabrication techniques will be employed in constructing future systems, for example, to handle the ever-increasing heat loads produced by advanced electronics while keeping the system as compact and light as possible.

Additive manufacturing has been demonstrated to produce a new material exhibiting a near-zero coefficient of thermal expansion (CTE) over an extended range of temperature $\left(\mathrm{CTE}=-0.22 \mathrm{ppm} /{ }^{\circ} \mathrm{C}\right.$ from $-196{ }^{\circ} \mathrm{C}$ to $\left.+24^{\circ} \mathrm{C}\right) .{ }^{17}$ The technique could someday enable fabrication of advanced light source components using materials with engineered properties, opening doors to the development of a new generation of light sources with the combination of high brightness and reduced footprint and cost.

\section{Acknowledgments}

The submitted manuscript has been created by UChicago Argonne, LLC, Operator of Argonne National Laboratory ("Argonne"). Argonne, a US Department of Energy Office of Science laboratory, is operated under Contract No. DE-AC02-06CH11357. The Department of Energy (DOE) will provide public access to these results of federally sponsored research in accordance with the DOE Public Access Plan. L.A. wishes to acknowledge M. White and A. Nassiri of ANL/APS for useful discussions and input on materials for accelerators and storage ring components.

\section{References}

1. E.J. Jaeschke, S. Khan, J.R. Schneider, J.B. Hastings, Eds., Synchrotron Light Sources and Free-Electron Lasers, Accelerator Physics, Instrumentation and Science Applications (Springer, Switzerland, 2016).

2. Z. Huang, "Brightness and Coherence of Sychrotron Radiation and FELS" (SLAC-PUB-15449), in Proc. 4th Int. Particle Accelerater Conf. (IPAC 2013), Shanghai, China, May 13-17, 2013, https://accelconf.web.cern.ch/accelconf/ IPAC2013/html/author.ht.

3. C. Jacobsen, M. Borland, Proc. Int. School Phys. "Enrico Fermi" 194, 35 (2016), doi:10.3254/978-1-61499-732-0-35.

4. C. Ji, A.F. Goncharov, V. Shukla, N.K. Jena, D. Popov, B. Li, J. Wang, Y. Meng, V.B. Prakapenka, J.S. Smith, R. Ahuja, W. Yang, H.-K. Mao, Proc. Natl. Acad. Sci. U.S.A. 114 (14), 3596 (2017).

5. M. Eriksson, J.F. van der Veen, C. Quitmann, J. Synchrotron Radiat. 21, 837 (2014).

6. L. Assoufid, T. Rabedeau, in Report of the Basic Energy Sciences Workshop on $X$-ray Optics for BES Light Source (Potomac, MD, March 27-29, 2013), https:// science.energy.gov/ /media/bes/pdf/reports/files/BES_XRay_Optics_rpt_print.pdf. 7. A. Aquila, R. Sobierajski, C. Ozkan, V. Hajkova, T. Burian, J. Chalupsky, L. Juha, M. Stormer, S. Bajt, M.T. Klepka, P. Dluzewski, K. Morawiec, H. Ohashi, T. Koyama, K. Tono, Y. Inubushi, M. Yabashi, H. Sinn, T. Tschentscher, A.P. Mancuso, J. Gaudin, Appl. Phys. Lett. 106, 241905 (2015).

8. E. Spiller, A.E. Rosenbluth, Opt. Eng. 25, 954 (1986).

9. P. Boher, L. Hennet, P. Houdy, Proc. SPIE 1345, 198 (1990).

10. I. Mohacsi, I. Vartiainen, B. Rösner, M. Guizar-Sicairos, V.A. Guzenko, I. McNulty, R. Winarski, M.V. Holt, C. David, Sci. Rep. 7, 43624 (2017).

11. R. Conley, N. Bouet, Y.S. Chu, X. Huang, H.C. Kang, A.T. Macrander, J. Maser E. Nazaretski, G.B. Stephenson, H. Yan, Synchrotron Radiat. News 29, 4 (2016). 12. Y. Chu, H. Yan, E. Nazaretski, S. Kalbfleisch, X. Huang, K. Lauer, N. Bouet, "Hard X-Ray Nanoprobe Facility at the National Synchrotron Light Source II," SPIE Newsroom (2015), doi:10.1117/2.1201508.006068.

13. T. Hatsui, H. Graafsma, Iucri 2, 371-383 (2015).

14. A. Grassellino, A. Romanenko, D. Sergatskov, O. Melnychuk, Y. Trenikhina A. Crawford, A. Rowe, M. Wong, T. Khabiboulline, F. Barkov, Supercond. Sci. Tech. 26, 10 (2013).

15. S. Posen, M. Liepe, D.L. Hall, Appl. Phys. Lett. 106, 082601 (2015).

16. A. Nassiri, R.L. Kustom, T. Proslier, T. Tan, X.X. Xi, in Proc. Supercond. Conf (Paris, France, September 22-27, 2013), http://accelconf.web.cern.ch/AccelConf/ SRF2013/papers/tup086.pdf.

17. W.A. Goodman, M.N. Ghasemi-Nejhad, S. Wright, D. Welson, in Material Technologies and Applications to Optics, Structures, Components, and Sub-Systems II, M. Krödel, J.L. Robichaud, W.A. Goodman, Eds. (SPIE, San Diego, 2015). 


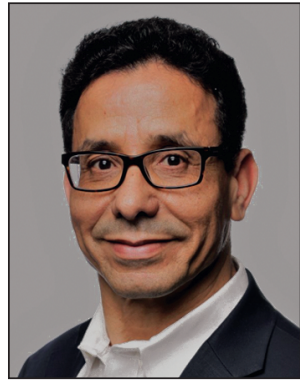

Lahsen Assoufid has been a group leader since 2011 of the Optics Group at the Advanced Photon Source (APS), a national x-ray synchrotron radiation user facility at Argonne National Laboratory. He obtained his $\mathrm{PhD}$ degree in physics from the Universite Paris Diderot, France, with dissertation research conducted at Cornell University. His prior experience at the APS included staff scientist, principal investigator for the X-ray Optics Metrology Laboratory, and section leader of the Mirrors, Multilayers, Optics, and Metrology Section of the Optics Fabrication and Metrology Group. He has organized numerous conferences and workshops in the field. $\mathrm{He}$ is a Fellow of OSA and SPIE. Assoufid can be reached by phone at 630-252 2774 or by email at assoufid@anl.gov.

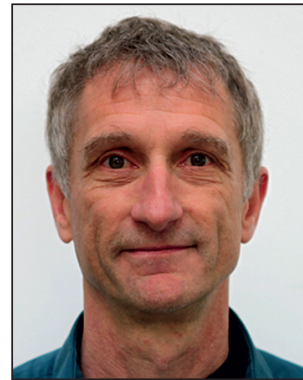

Heinz Graafsma has been head of the PhotonScience Detector Group at Deutsches ElektronenSynchrotron, Germany, a research center for photon science, accelerator physics, and particle and astro-particle physics since 2006. He obtained his PhD degree in applied physics from the University of Twente, The Netherlands, and the State University of New York at Buffalo, based on studies of crystals in electric fields using synchrotron radiation at the National Synchrotron Light Source at Brookhaven National Laboratory. Prior to this, he was a beamline scientist and head of the Instrumentation Group at the European Synchrotron Radiation Facility, France. Graafsma can be reached by phone at $+49(0) 408998$ 1678 or by email at heinz.graafsma@desy.de.

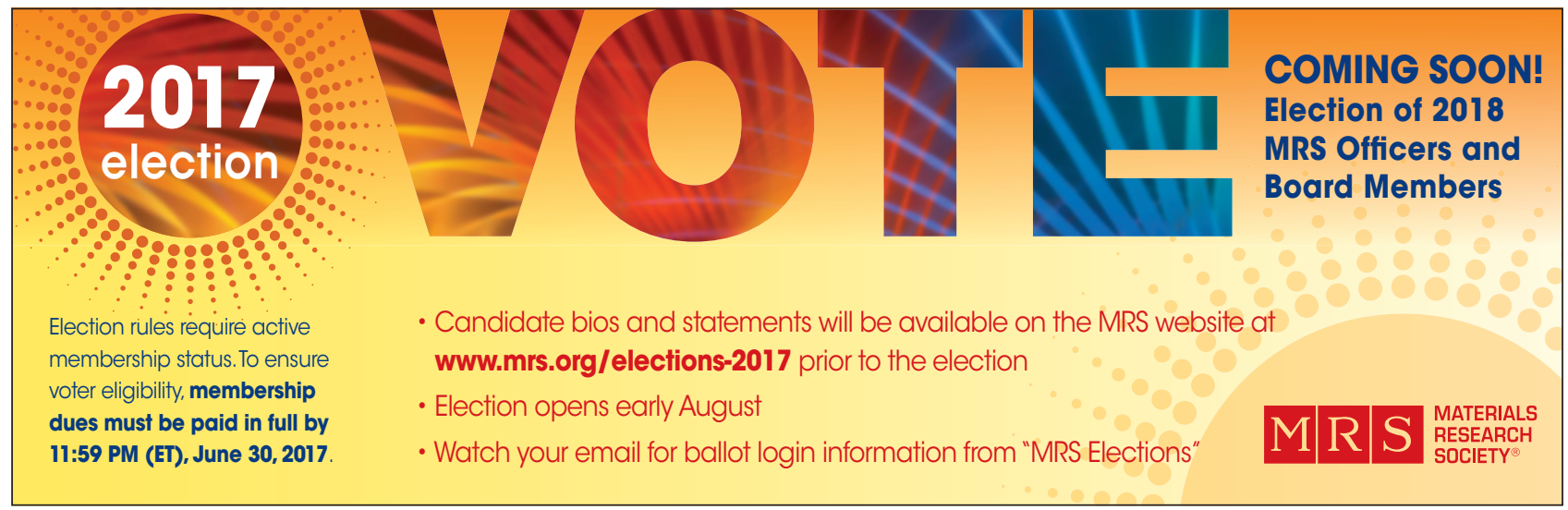

\section{High Resolution RBS}

National Electrostatics Corporation has added Ångstrom level, High Resolution RBS to the RC43 Analysis System for nanotechnology applications. A single Pelletron instrument can now provide RBS, channeling RBS, microRBS, PIXE, ERDA, NRA, and HR-RBS capability, collecting up to four spectra simultaneously.

Pelletron accelerators are available with ion beam energies from below $1 \mathrm{MeV}$ in to the $100 \mathrm{MeV}$ region.

Www.pelletron.com

Phone: 608-831-7600

E-mail: nec@pelletron.com

\section{National Electrostatics corro:}

